\title{
Clinical concerns associated with cannabis legalization
}

\section{Keywords: cannabis, legalization, toxin, chemistry, neurobiology \\ Introduction \\ Which way will the wind blow?}

As the wildfire of cannabis legalization rages across the continent, it is not at all clear whether adolescentswill benefit or get burned. Will medicinal marijuana be a tonic, or a toxin? In order to shed some light on the question, we undertook a review of the recent literature, media and structured analysis of patient narratives.

\section{Cannabis usage}

The Substance Abuse and Mental Health Services Administration (SAMHSA) reports that 7.3 percent of Americans, almost 20 million people reported using marijuana in the last year. More to the point, $40 \%$ of adolescents 12-18 years old reported using cannabis at least once in their lifetime and $10 \%$ reported use in the past year.

\section{Youth Views on cannabis}

Historical shifts are occurring in attitudes toward marijuana. In 2015, the proportion of adolescents who perceive great risk of harm in using marijuana once or twice a week has decreased from 55\% in 2007 to $44 \%$ in 2012 . $^{2}$

When questioned, the critical mass of teen users justifies their use of cannabis based on a number of strongly held beliefs:
a. not harmful
b. beneficial medicinal effects
c. not addictive
d. not associated with withdrawal

\section{Myth: cannabis is not harmful}

Specifically, adolescents' general perception is that cannabis is no more harmful or addictive than other recreational drugs, such as alcohol or nicotine. These sentiments are echoed by advocates for legalization who point out that the morbidity, mortality, and economic costs to society, associated with alcohol and tobacco use, dwarf those associated with marijuana use. ${ }^{3}$ Associations between Cannabis Use and Physical Health Problems in Early Midlife: A Longitudinal Comparison of Persistent Cannabis vs Tobacco Users. ${ }^{4}$

In support of this, Monitoring the Future, a cross-sectional survey of more than 250 thousand American high school students, shows that adolescents' perception of the harmfulness of cannabis deceased from 2010 to 2015. More importantly, in lockstep with a decrease in perceived harmfulness, is an increase in use in that same population. However, it is not that simple. This increase in marijuana use following recreational marijuana legalization was seen in Washington but not in Colorado.

Prevalence of marijuana use does not differentially increase among youth after states pass medical marijuana laws: Commentary on and reanalysis of US National Survey on Drug Use in Households data $2002-2011.5$
Volume 5 Issue 4 - 2018

\author{
Anthony Ocana \\ Specialist in Addiction Medicine, USA
}

Correspondence: Anthony Ocana, Specialist in Addiction Medicine, North Vancouver, BC North Shore ADHD and Addiction Clinic, Email aocana@me.com

Received: February 15, 2018| Published: August 23, 2018

In 1995, very early in the discussion of legalization, proponents of legalization got the medical endorsement they were waiting for when the editors of The Lancet concluded that "cannabis, per se, is not a hazard to society but driving it further underground may well be." Deglamorizing Cannabis [editorial]. Lancet.1995,346:1241.

However, if the discussion is to be clinically relevant, we must first be clear that we are NOT talking about the benefits of cannabis legalization to society, nor the relative harm of cannabis compared to other legal drugs. Similarly, our concern is not with perceived harm, but rather with the actual harm of cannabis use. The relevant data in this case must inform the harm to the most vulnerable cohort: those with known mental health and addiction co-morbidity, who are heavy users.

I propose that besides adolescents, 13-18 years old, we include young adults. This second group is included because neurobiologically, their brains are still developing. Specifically, thecircuits governing judgment and self-inhibitionwhich continue to mature from 19-25 years old. According to Nora Volkov, director of NIDA, heavy cannabis use compromises cognitive development, impairing adolescents' ability to crucial emotional, academic and social milestones.

The other reason to include adolescents with young adults is to avoid misreading the data. Specifically, these two groups usage will differ as the effects of cannabis legalization are played out in practical terms. For example, the most recent data from the National Survey on Drug Use and Health shows that following legalization in 2014, the rate of adolescent marijuana use, among 13-18-year-olds in Colorado fell for the subsequent two years, to its lowest level in nearly a decade.

This data has recently been cited by proponents of legalization as a marker that the anticipated harms are overblown. But these data refer to the younger cohort of 13-18-year olds, where legalization has actually brought more regulation, oversight and controlled access. "Teen use appears to be dropping now that state and local authorities are overseeing the production and sale of marijuana," said Brian Vicente of Vicente Sederberg LLC, one of the drafters of Colorado's marijuana ballot measure, in a statement. "There are serious penalties for selling to minors, and regulated cannabis businesses are being vigilant in checking IDs."

On the other hand, cannabis use in the older cohort, those 19-25years-oldhas actually increased. And it is the increased use these 
young adults that pose the greatest cost to society. The only way that there can be any discussion of the benefits of cannabis legalization is to also know the dangers. And if we were to make such a comparison, we would have demonetized costs of cannabis induced adverse outcomes.

\section{Understanding cannabis}

If we are to have any defense against the potential tsunami of increased cannabis-induced adverse outcomes, we need to understand the factors that drive cannabis use in the first place so that we can create a risk mitigation strategy. Why is this behavior so sticky? What's the upside for youth to use cannabis in the first place? to clarify these muddy waters, we invited 100 patients, 16 to 25 yearsold, who smoke cannabis more than 20 days/ month, to speak openly about their rationale for using cannabis and the perceived benefits.

We used qualitative content analysis, using a standard approach (Forman and Dam-Schroder, 2008), coding and organizing patients' responses to standardized questions using QSR NVivo 11 for Mac. Responses are transcribed and hand-coded by two researchers. The hypotheses are refined to incorporate re-occurring themes that emerge from the data themselves.

While these narratives are by definition anecdotal evidence, NVivo is widely used narrative analysis software, used in the quantification of behavioral health data, and yields scholarly work found in peer-review publications and scientific communications. If certain responses occur over and over, independently, the pattern can ethically be used to inform our understanding of adolescent cannabis use.

\section{Know your dope!}

One of the re-occurring themes in patients' narratives is their very specific modulation of various factors in order to get the desired therapeutic effects:

a. Strain of cannabis (Sativa vs. Indica)

b. Day-time or evening

c. Amount

\section{Cannabis botany}

Despite the huge variety of marijuana available these days, almost all of it (over 99\%) is ultimately derived from only two species: Cannabis sativa and Cannabis Indica. This distinction is crucial as the two strains have radically different chemical composition and medicinal properties: Sativa stimulates; Indica sedates.

Sativa come from Colombia, Mexico, Thailand and Southeast Asia. "Sativa" and its descendants came originally from Pakistan and Afghanistan. Sativa dominant strains are tall, thin plants, with much narrower leaves and grow a lighter green in color.

"Indica" refers to plants of Indian heritage. Indices originally come from the hash producing countries of the world like Afghanistan, Morocco and Tibet. Indica dominant strains are short dense plants, with broad leaves and often grow a darker green. While Extensive cross-breeding has entangled the strains over the years, phytochemical, genetic and clinical research still supports their separation.

\section{Cannabis chemistry}

Sativa dominant strains are higher in Cannabinol (THC). Results from a drug detection laboratory indicated that the average of $10 \mathrm{C}$. sativa measured: THC $25 \%$, CBD $<0.1 \%$ and $\mathrm{CBN}<0.1 \%$. Indica dominant strains are higher in Canadian (CBD). Results indicated that C. Indica strains averaged THC $20 \%, \mathrm{CBD}<0.2 \%$ and $\mathrm{CBN}<0.5 \%$.

Hybrids will vary in their composition of THC, CBD and other cannabinoids, and are often referred to based upon the dominant cannabinoid ratio inherited from theirlineage. Cultivators and distributors often give some of these varieties more personal names, such as Acapulco Gold, Panama Red, Northern Lights. Chemical constituents of marijuana: the complex mixture of natural cannabinoids. ${ }^{6}$

\section{Cannabis neurobiology}

Cannabinoids are basically derived from three sources:

(a) Phytocannabinoids are cannabinoid compounds produced by plants Cannabis sativa or Cannabis indica

(b) Endocannabinoids are neurotransmitters produced in the brain or in peripheral tissues, and act on cannabinoid receptors

(c) Synthetic cannabinoids, synthesized in the laboratory, are structurally analogous to phytocannabinoids or endocannabinoids and act by similar biological mechanisms. ${ }^{7}$

In very simplified terms, consumption of cannabis interacts with at least two known cannabinoid receptors:

a. Stimulation of CB1 receptors, densely distributed predominantly throughout the brain producing a variety of hallucinogenic and stimulant effects.

b. CB2 stimulation of immune tissues and cells in the periphery is associated with pain relief, sedation and anxiolytics.

Cannabinoids exert their psychoactive effects by modulating neurotransmission in multiple areas including peripheral serotonergic, mesolimbic dopaminergic circuits, GABAergic and Glutamatergic circuits. It is the increase in GABA and the decrease in Glutamate neurotransmission that gives Indica its mood-stabilization effects. ${ }^{8}$

In our patient's narratives, treatment with glutamate blockers such as Lamotrigine, decreased the agitation and hyper-cognition associated with cannabis withdrawal.

\section{Beneficial medicinal effects are strain-specific}

Some synthetic cannabinoids have approved therapeutic applications. For instance, the psychoactive cannabinoid, THC (e.g., within Marinol $($ ) $)$, has approval for either its anti-emetic and appetite stimulating properties or as a treatment for multiple scelerosis in Canada, Denmark and the United States, and Sativex ${ }^{\circledR}$, a combination of THC and CBD, has approval for spasticity in 25 countries. But most adolescents do not have access to synthetic cannabinoids. They also do not specifically use THC or CBD as is used in most research studies. Adolescents in our practice almost exclusively smoke dried cannabis that they may get legally from dispensaries, but more commonly, illegally through established channels. According to our patients, Sativa strains are chosen for their ability to stimulate: lift mood and improve cognitive and executive function.

Indica strains are experienced as sedating: improving sleep, relaxing muscles, relieving pain and assuaging anxiety. Indica is also known for its anti-nausea, anti-seizure properties. Here is the narrative of a 19-year-old daily cannabis user. It is typical of the cohort and uncovers a number of previously unappreciated patterns and trends. 
"I smoke Sativa during the day, it is more stimulating and it helps me get things done. I smoke Indica before bed. It relaxes my muscles and eventually helps me sleep. Psychologically, it slows down my thinking process and I feel subtly happier, and calm. The problem is, I wake up in a daze and I need a coffee to get out the door."

\section{Practical implication}

These two strains have radically different chemical composition and medical properties. The neurobiological effects of the two strains are essentially opposite. Therefore, it behooves any clinician who deals with adolescents to know the difference (Figure 1) (Figure 2).

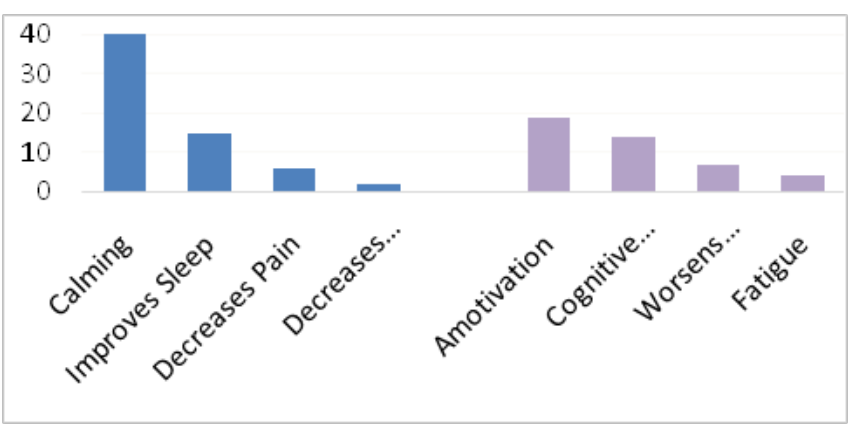

Figure I Number of references coded from 100 patient's narratives.

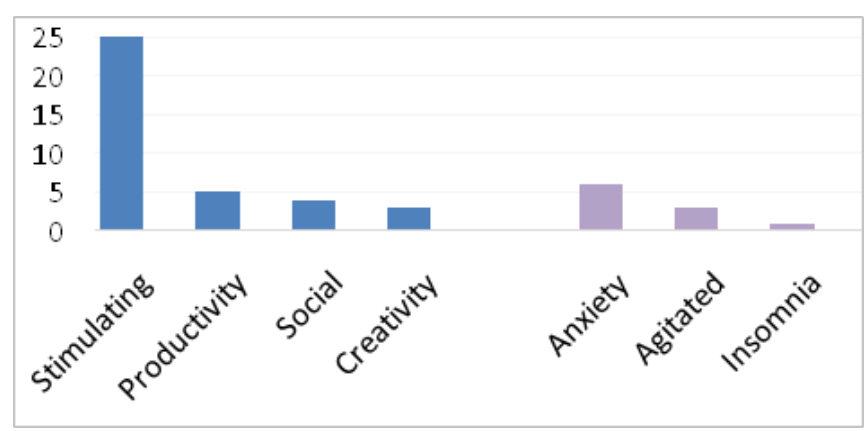

Figure 2 Number of references coded from 100 patient's narratives.

\section{Acute risks of cannabis}

\section{The impact of cannabis legalization will be greatest in the ER}

Let's face it. Adolescents smoke a lot of marijuana. For the most part this proceeds without any obvious incident. One of the problems to prove otherwise is that evidence has a piecemeal approach to reporting of young adult sequelae and existing data are limited in statistical power to examine rarer outcomes and less common, heavier patterns of cannabis use than those already investigated. ${ }^{9}$ However, research presented at the 2017 Pediatric Academic Societies Meeting in San Francisco suggests that the more significant impact of legalization may be in the ER.

Dr. Wang, an assistant professor of pediatrics at the University of Colorado Anschutz Medical Campus, reviewed the hospital system's emergency department and urgent care records for 13 to 21 -year-olds seen between January 2005 and June 2015. They found that the annual number of visits with a cannabis related diagnostic code or positive for marijuana from a urine drug screen more than quadrupled during the decade, from 146 in 2005 to 639 in 2014.

\section{Cannabis overdose}

As with any substance, the devil is in the dosage. Cannabis overdose is more common in novice users who underestimate its potency. The symptoms include nausea, vomiting, suspicion/paranoia, agitation and panic, not uncommonly psychosis and occasional respiratory depression that in combination with other drugs can be life-threatening.

Edibles probably pose the greatest risk to the inexperienced. Edibles are deceptive. And, by their very nature, are more dangerous because they have a slower onset and longer lasting effects.says Dr. Richard Zane, Emergency department head at University of Colorado Hospital.

It is impossible to gauge the potency of an edible and even harder to predict the time of onset. Inexperienced users may eat too much or not wait long enough for it to take effect before having more. Some edibles are marketed as candy in the shape of lollipops or gummy bears leaving younger users unprepared for their very serious effects. This is manifested as a nearly threefold increase in pediatric and adolescent ED visits. ${ }^{10}$

\section{Cannabis induced psychosis}

Of all the adverse effects of cannabis overdose, the most dangerous is psychosis. In order to gain some insight into the risk factors. We reviewed the charts of 12 patients who, were admitted to hospital, between 2016 and 2017 with cannabis-induced psychosis. The common narratives associated with it were as follows:

1. The inexperienced user miscalculating the amount or potency of cannabis products

2. $100 \%$ of psychotic admissions were associated with Sativa

3. A history of schizophrenia or previous drug-induced psychosis

4. Self-reported dehydration or recent sleep debt

5. The presence of other stimulants at the time of consumption.

Here is the transcript of an 18-year-old freshman who was apprehended by the police, taken to the ER, and released into the custody of his parents. "I was taking my friend's Adder all XR to help me study. I was really stressed during exams and not getting much sleep. The next day, before the exam, I had an energy drink and smoked a Sativa to perk me up.

I was found by police, darting through the forest behind campus, thinking I was being followed and that someone had hacked into my computer.

My parents came to drive me home, and on the way, I jumped out of the car because I was convinced that there was a bomb in my mother's stomach."

\section{Practical implication}

The data suggest that the combination of Cannabis sativa plus any other stimulants made for the most explosive outcomes and likely describes the tipping point, in the short-term at least, at which cannabis becomes a toxin. Physicians should be aware of these factors and be prepared to educate their adolescent patients to ways to manage the risk of psychosis associated with cannabis use. 


\section{Cannabis addiction}

Experimental Cannabis use won't set an irreversible course for addiction. However, a review of recent epidemiological data from various countries cans fam Physician. Suggest the following broad patterns among youth and young adults who experiment with cannabis:

I. $40-50 \%$ enjoy the experience

II. $25-30 \%$ become recreational users

III. $15-20 \%$ experience withdrawal and continue to use regularly

IV. $5-10 \%$ become daily users

There is definitely a withdrawal effect seen in chronic daily users of high potency products who stop consuming suddenly. ${ }^{11}$

The associated dysphoria often prompts a return to continued use, despite negative consequences, the de-facto definition of addiction.

If we use the experience of withdrawal as a proxy for the addiction potential of cannabis, there are two principle factors at play:

I. Increased Fat solubility decreases withdrawal

II. Increased potency increases withdrawal

Cannabinoids are the only drug of abuse that is fat soluble and accumulates in the body. Specifically, the rate of decline of active metabolites is significantly slower than alcohol, nicotine, stimulants, cocaine or opiates. Therefore, the withdrawal from cannabis would be expected to be slower and may explain why it is experienced as less addictive.

\section{Cannabis potency}

The potency of cannabis has increased markedly in the last 40 years. The pot smoked by today's adolescents is much higher than what their parents used in the $60 \mathrm{~s}$ and $70 \mathrm{~s}$, when it was $2-3 \%$ THC. In 2015, in Colorado's legal cannabis, the average THC level was 18.7 percent, and some retail pot contains 30 percent THC or more.

Pot evolution: how the makeup of marijuana has changed over time. ${ }^{12}$

Simply put, higher potency cannabis is more adverse events, especially by inexperienced users. It is associated with more withdrawal and by extension, increased risk of addiction. Here is a typical narrative from a daily cannabis user:

"When I cut pot out completely, I became more anxious and found that I could not sleep and that bothered me. When I started smoking again, I felt better, but then I felt like I was addicted and that's not what I really wanted."

\section{Long term risks of cannabis}

The most sinister outcomes associated with cannabis are the longterm effects on mood, motivation and mental performance. However, interpreting the literature is complicated by a number of factors:

i. difficulty to identifying causality

ii. lack of strain specificity (Sativa and Indica have essentially opposite effects)
This creates noise in the data and results in amusingly contradictory findings. ${ }^{13}$

\section{Cannabis and cognition}

On the one hand medical experts see cannabis (non-specifically) as imperceptible decreasing IQ and cognitive function, correlated to higher rates of academic failure and lower vocational attainment. ${ }^{14}$ In contradiction, despite no clinical recommendations or systematic research supporting the beneficial effects of cannabis use for ADHD, online discussions indicate that cannabis (Sativa) is considered therapeutic for ADHD. And of course, both are correct. ${ }^{15}$

\section{Cannabis and depression}

Similar confusion is seen in the association between cannabis and mood disorders. Many studies did not indicate any overall pattern of excess psychopathology associated with (non-specific) cannabis use, although significant differences were found in levels of depression. However it is not clear whether impaired motivation is a manifestation of depression, rather than a consequence of frequent heavy use. ${ }^{16}$

There are differing views, as expected. In participants with no baseline depressive symptoms, those with a diagnosis of cannabis abuse at baseline were four times more likely than those with no cannabis abuse diagnosis to have depressive symptoms at the follow-up assessment, after adjusting for age, gender, antisocial symptoms, and other baseline covariates. ${ }^{17}$

In our own patients' narratives, there were a number of patterns repeatedly found regarding their strain-specific experiences (see above)

\section{Sativa}

i. Improves mood acutely

ii. But in excess, increases anxiety, sometimes triggering mania or psychosis

\section{Indica}

a. Decreases anxiety, cognitive hyperactivity, pain and nausea

b. But in excess, decreases same day motivation and productivity

c. Decreases long term self-efficacy

d. Increased social isolation

e. Increased depressive symptomatology

f. Lower academic and vocational attainment

\section{Where do we go from here?}

My sense is that, in the tradeoff between the stabilization of the cannabis trade and youth mental health, youth will lose. When the legalization-driven increase in accessibility and use of high potency smoked and edible cannabis, combines with adolescent misadventure, the resultant tsunami will plow into families, educational institutions and the ER, with predictably negative outcomes. Moreover, it seems to me that we are already ill-prepared for managing youth cannabis abuse. Will we be for more? 
There are certainly not enough physicians trained in mental health and addiction, nor evidence-based programs dedicated to this demographic, therefore I would suggest that investment in evidencebased adolescent substance use prevention and treatment programs should be mandatory in jurisdictions that legalize recreational marijuana use.

Media campaigns that educate youth on the short and long-term risks of using cannabis, while their brains are still developing will need to be established. Building capacity and resources for parents to discuss cannabis use with their adolescent children will be needed to provide guidance as communities navigate the new landscape of legal recreational marijuana. ${ }^{18}$

\section{Summary}

a) It is our adolescent patients' experience that cannabis helps them cope with a number of mental and physical conditions.

b) Daily users specifically manipulate the strain, the dosage and the timing to achieve their desired effects.

c) There are two stains of cannabis: Indica and Sativa, that have essentially opposite effects.

d) Sativa is stimulating and increases cognitive and executive function. It is more likely consumed in the day-time. On the negative side, it can over-stimulate and is associated with agitation and not uncommonly, with psychosis.

Indica is generally perceived as sedating, improves sleep, decreases pain and agitation. It is preferentially consumed in the evening. On the negative side, it can over-sedate and is associated with next-day amotivation and cognitive impairment.

\section{Acknowledgements}

None.

\section{Conflict of interest}

Author declares there is no conflict of interest.

\section{References}

1. Results from the 2012 National Survey on Drug Use and Health: Summary of National Findings, NSDUH Series H-46, HHS Publication No. (SMA) 13-4795. Rockville, MD: Substance Abuse and Mental Health Services Administration, 2013.

2. Jones JM Gallup. [Accessed February 15, 2018];US, 58\%back legal marijuana use.

3. Cerdá M, Moffitt TE, Meier MH, et al. Persistent cannabis dependence and alcohol dependence represent risks for midlife economic and social problems: A longitudinal cohort study. Clin Psychol Sci. 2016;4(6):10281046 .
4. Meier MH, Caspi A, Cerdá M, et al. Associations Between Cannabis Use and Physical Health Problems in Early Midlife: A Longitudinal Comparison of Persistent Cannabis vs Tobacco Users. JAMA Psychiatry. 2016;73(7):731-740.

5. Wall MM, Mauro C, Hasin DS, et al. Prevalence of marijuana use does not differentially increase among youth after states pass medical marijuana laws: Commentary on and reanalysis of US National Survey on Drug Use in Households data 2002-2011. Int J Drug Policy. 2016;29:9-13.

6. Elsohly MA, Slade D. Chemical constituents of marijuana: the complex mixture of natural cannabinoids. Life Sci. 2005;78(5):539-548.

7. Update of Cannabis and its medical use-World Health Organization.

8. Rigucci S, Xin L, Klauser P, et al. Cannabis use in early psychosis is associated with reduced glutamate levels in the prefrontal cortex. Psychopharmacology (Berl). 2018;235(1):13-22.

9. Edmund Silins, L John Horwood, George C Patton. Young adult sequelae of adolescent cannabis use: in integrative analysis. Lancet Psychiatry. 2014;1:286-293.

10. Year 1 of Legal Marijuana: Lessons Learned in CO. By R. Scott Rappold, WebMD Health News.

11. Greene MC, Kelly JF. The prevalence of cannabis withdrawal and its influence on adolescents' treatment response and outcomes: a 12-month prospective investigation. $J$ Addict Med. 2014;8(5):359-367.

12. Pot evolution: how the makeup of marijuana has changed over time.

13. Gonzalez R. Acute and Non-acute Effects of Cannabis on Brain Functioning and Neuropsychological Performance. Neuropsychol Rev. 2007; 17(3):347-361.

14. Brook JS, Lee JY, Finch SJ, et al. Adult work commitment, financial stability, and social environment as related to trajectories of marijuana use beginning in adolescence. Subst Abus. 2013;34(3):298-305.

15. John Mitchell T, Maggie M, Sweitzer, et al. "I Use Weed for My ADHD": A Qualitative Analysis of Online Forum Discussions on Cannabis Use and ADHD. PLoS One. 2016;11(5):e0156614.

16. Lac A, Luk JW. Testing the A motivational Syndrome: Marijuana Use Longitudinally Predicts Lower Self-Efficacy Even After Controlling for Demographics, Personality, and Alcohol and Cigarette Use. Prev Sci. 2018;19(2):117-126.

17. Bovasso GB. Cannabis abuse as a risk factor for depressive symptoms. Am J Psychiatry. 2001;158(12):2033-2037.

18. Julie Rusby C, Erika Westling, Ryann Crowley, et al. Legalization of Recreational Marijuana and Community Sales Policy in Oregon: Impact on Adolescent Willingness and Intent to Use, Parent Use, and Adolescent Use. Psychol Addict Behav. 2018;32(1):84-92. 